\title{
Chronisch obstruktive Lungenerkrankung
}

\section{Nichtberichten von Exazerbationen schadet der Lebensqualität}

Xu W et al. Negative impacts of unreported COPD exacerbations on health-related

quality of life at 1 year. Eur Respir J. 2010;35:1022-1030

\section{Hintergrund und Fragestellung}

Häufig berichten Patienten mit einer chronisch obstruktiven Lungenerkrankung (COPD) ihrem Arzt aufgetretene Exazerbationen nicht. Das Ziel der vorliegenden Arbeit war es, den mittel- bis langfristigen Einfluss der nicht berichteten Exazerbationen auf die Lebensqualität von COPD-Patienten zu untersuchen.

\section{Methodik}

Die multizentrische prospektive Kohortenstudie umfasste 491 chinesische COPD-Patienten. Die gesundheitsbezogene Lebensqualität wurde mithilfe des St. George's Respiratory Questionnaire (SGRQ) bestimmt. Andere Erhebungen beinhalteten klinische, psychosoziale, soziodemografische und therapeutische Daten. Die Patienten wurden zwölf Monate lang einmal pro Monat nach Exazerbationen (mindestens ein sich verschlechterndes COPDLeitsymptom, das länger als 48 Stunden bestand) befragt.

\section{Ergebnisse}

466 nicht berichtete und 410 berichtete Exazerbationen wurden dokumentiert. Während dieses einen Jahres mussten $30 \%$ der Patienten aufgrund mindestens einer Exazerbation hospitalisiert werden. 87\% der Patienten mit berichteten Exazerbationen wurden mit Antibiotika, Steroiden und/oder Bronchodilatatoren behandelt. 20\% der Patienten mit nicht berichteten Exazerbationen behandelten sich mit Medikamenten aus Restbeständen oder frei verkäuflichen Medikamenten selbst.

Die Veränderung der Gesamtpunktzahl im SGRQ war bei Patienten mit lediglich einer nicht berichteten Exazerbation ähnlich der Gesamtpunktezahl bei Patienten ohne Exazerbation (adjustierte mittlere Veränderung: 1,22 Punkte; 95\%-Konfidenzintervall: 4,05-6,48), während sich bei Patienten mit mehr als einer nicht berichteten Exazerbation eine signifikant schlechtere Lebensqualität zeigte (4,61 Punkte; 95\%-Konfidenzintervall: 0,09-9,13).

\section{Schlussfolgerung}

Die Entwicklung von Selbstmanagementprogrammen mit dem Fokus auf der frühzeitigen Erkennung einer COPDExazerbation und die konsequente Umsetzung eines entsprechenden Aktionsplans könnten sinnvoll sein.

\section{Kommentar}

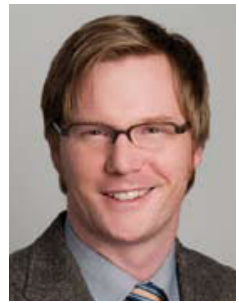

Dr. med.

Felix C. Ringshausen, Zürich

In dieser Arbeit hatten nicht berichtete COPD-Exazerbationen einen bedeutsamen negativen Einfluss auf die gesundheitsbezogene Lebensqualität. Die Ergebnisse verdeutlichen das Potenzial, das die Aufklärung und die Anleitung von COPD-Patienten zum frühzeitigen Selbstmanagement einer Exazerbation haben könnten.

Zukünftige klinische Studien sollten sowohl die Verbesserung der Lebensqualität als auch die Häufigkeit der nicht berichteten Exazerbationen als einen wichtigen Endpunkt mit erheben. Zunächst sollten jedoch die Durchführbarkeit und vor allem die Sicherheit eines abgestuften Selbstmanagement-Programms von COPD-Exazerbationen über einen längeren Beobachtungszeitraum belegt werden. 